\title{
Artificial pancreas techniques based on robust model predictive controller
}

\author{
Waleed Khalid Al-Azzawi \\ Faculty of Information Technology, Arab Open University Bahrain, Iraq
}

\begin{tabular}{|c|c|}
\hline Article Info & ABSTRACT \\
\hline & \multirow{5}{*}{$\begin{array}{l}\text { Diabetes is known as the major cause of death in the world leading to kidney, } \\
\text { retinopathy and cardiovascular diseases as well. In this paper, a Robust } \\
\text { Model Predictive Controller (RMPC) is introduced to design artificial } \\
\text { pancreas that solved the model uncertainty and keep the blood glucose level } \\
\text { in the normal range by regulating the size of insulin infusion from pump } \\
\text { based on RMPC. The simulation results will present a good performance of } \\
\text { the proposed controller to avoid disturbance and robustness against } \\
\text { uncertainties. }\end{array}$} \\
\hline Received May 3, 2019 & \\
\hline Revised Jul 5, 2019 & \\
\hline Accepted Aug 1, 2019 & \\
\hline Keywords: & \\
\hline
\end{tabular}

Artificial pancreas

MPC techniques

Robust model predictive

controller (RMPC)

Copyright $\odot 2019$ Institute of Advanced Engineering and Science. All rights reserved.

\section{Corresponding Author:}

Waleed Khalid Al-Azzawi,

Faculty of Information Technology,

Arab Open University Bahrain, Iraq.

Email: waleedki@yahoo.com

\section{INTRODUCTION}

Type 1 diabetes is an inveterate disease in which the pancreas does not produce sufficient insulin to control glucose levels in the blood. In the shortage of insulin, the admittance of glucose into skeletal, cardiac, smooth muscle and other tissues is reduced. When insulin is missing for a longer period of time, the muscle and tissue cells will start using fat as energy source, in place of glucose from the blood stream. The disturbance of meal eating presents great challenges to automatic blood glucose control. There are many factors must be measured to regulate blood glucose such as. Fats and proteins cause delays in absorption of glucose from carbohydrates eaten at the same time, physical exercise affects the blood glucose regulation by reducing the requirement for insulin, and furthermore, stress and physical illness are involved in the blood glucose regulation process. Thus, designing accurate predictive models are hard to achieve. In 2011, Dimitri Boiroux et al, applied a robust feedforward-feedback control strategy to people with type 1 diabetes. The feed forward controller consists of a bolus calculator which compensates the disturbance coming from meals. The feedback controller is based on a linearized description of the model describing the patient [1].

In 2008, Gianni Marchetti et al concerned with the improvement of new feed forward-feedback control techniques for actual glucose control and type 1diabetes. Enhanced post-meal responses can be achieved by a pre-prandial snack or bolus, or by reducing the glucose set-point prior to the meal [2]. In 2017, Mojgan Esna-Ashari et al proposed a technique for blood glucose level regulation in type I diabetes. The control approach is based on non-linear model predictive control. The purpose of the proposed controller optimized with genetic algorithms is to measure blood glucose level every time and estimate it for the next time interval [3]. In 2017, Lukas Ortmann et al, introduced a Model Predictive Controller that takes the periodic Insulin sensitivity into account, so as to improve blood glucose control. The upcoming effect of the Insulin sensitivity is estimated by a machine learning technique, namely, a customized Gaussian Process (GP), based on historical training data [4]. In 2017, Chiara Toffanin, proposed an adaptive Model Predictive 
Control approach to account for it and test it in silico. Run-to-Run method adapts the subcutaneous basal insulin delivery throughout the night and the carbohydrate-to-insulin ratio throughout the day, according to some performance indices measured from subcutaneous continuous glucose sensor data [5]. In 2017, Berno J. E. Misgeld et al presented a novel method towards real-time estimation of insulin sensitivity in type-1 diabetes. The predictive of insulin sensitivity is investigated while a carbohydrate uptake disturbance takes place simultaneously [6]. In construct, in this paper, the Robust Model Predictive Controller (RMPC) design approach will be employed for the regulation of constrained systems. RMPC algorithms are able to coordinate hard constraints on state outputs and inputs. Stability and robustness are considered properties of RMPC controllers, as they are essential to hardware implementation. A whole theory on robust stability of RMPC has been applied for linear and nonlinear systems.

\section{PROBLEM DESCRIPTION}

The controllers are built in both traditional and RMPC. However, it holds only for linear scheme of the system. While dealing with the non-linear system operations, the system should be linearised. This linearised form of the non-linear system could be properly control using traditional controllers such as (i.e. Model Predictive Controller). In an Artificial Pancreas regulation, continuous subcutaneous insulin infusion common with subcutaneous glucose sensing has been shown to be effective for feed forward channel "out-of meal" periods, preservation blood glucose in the regular level in the post absorptive case. However, the feedback channel has not been so successful in treating insulin desires at meals state [7, 8]. In spite of developments in sensor and pump equipment, the Artificial Pancreas must coordinate with the delays and inaccuracies in glucose sensing. This is very hard when a system disturbance, such as, a meal, happens and triggers a fast glucose increase that is considerably faster than the time required for insulin absorption. The stability problem of constraints plants in closed-loop with MPC controllers is shown in Figure 1.

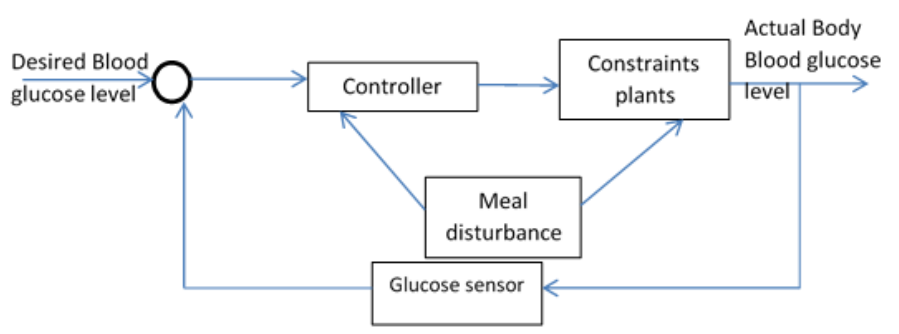

Figure 1. Artificial pancreas techniques

\section{METHODOLOGY}

Model predictive control is a method of control in which the present control signal is obtained by solving, at each sampling time, a finite constraints open-loop optimal control problem, using the recent state of the system as the initial state, the optimization produces an optimal control signal sequence and the first control signal in this sequence is applied to the system. MPC is able to cope with hard constraints on control signals and states as shown in Figure 2. Therefore, been applied in medical application where approval of constraints is mostly very significant since efficiency requests operating points on or close to the limit of the set of acceptable states and control signals.

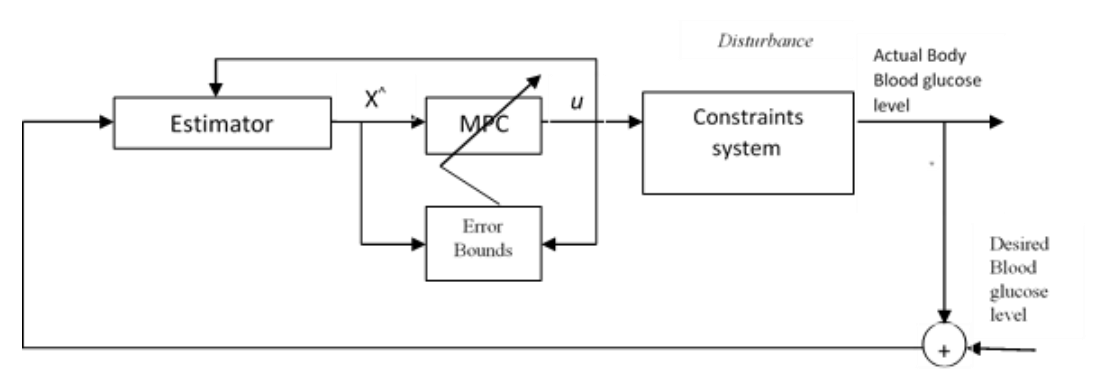

Figure 2. MPC for constraints system 


\section{MODELING AND SIMULATION}

In this paper, the MPC design will be employed for the regulation of constrained systems. A robust stability of MPC has been avoid violations of input and output constraints drive certain output variables to their optimal set points, while keeping other outputs within specified value. Avoid redundant movement of the input variables. Estimated values of output variables are predicted using a linear quadratic regulator of the plant and current measurements. The control signal calculations are according to both upcoming predictions and recent measurements. The control signalsu $(k)$, at the $k^{\text {th }}$ sampling time are calculated so that they minimize a performance index J. the constraints of optimal control system as (1):

$$
\left\{\begin{array}{l}
u(k)_{\text {min }} \leq u(k) \leq u(k)_{\text {max }} \\
y(k)_{\text {min }} \leq y(k) \leq y(k)_{\text {max }}
\end{array}\right.
$$

The Insulin kinetic equations are (2):

$$
\begin{aligned}
& \dot{I}=\frac{1}{T_{x}}\left[-I(t)+k_{i} S(t)\right] \\
& \dot{X}=\frac{1}{T_{m}}[-X(t)+I(t)] \\
& \dot{S}=\frac{1}{T_{i}}[-S(t)+U(t)]
\end{aligned}
$$

Where, I- insulin plasma, X- insulin interstitial, S- insulin sensitivity and U- control signal While, the glucose kinetic equations are (3) [9]:

$$
\begin{aligned}
& \dot{G}=\frac{G(t)}{T_{y}}+\frac{Y(t)}{T_{G}}+\frac{1}{U_{G}}\left[E_{g}(t)+E_{b}(t)\right] \\
& \dot{Y}=K_{y}\left[\frac{G(t)}{T_{y}}-\frac{Y(t)}{T_{G}}\right]-k_{i} X(t) Y(t)
\end{aligned}
$$

where, G- glucose plasma, and Y- subcutaneous compartment.

According to medical experience $T_{x}, k_{i}, T_{m}, T_{i}, T_{y}$, and $T_{G}$ can be considered constant. According to insulin and glucose kinetic equation the state space representation for both insulin and glucose equations are $(4-5)$ :

$$
\begin{aligned}
& {\left[\begin{array}{c}
\dot{I} \\
\dot{X} \\
\dot{S}
\end{array}\right]=\left[\begin{array}{ccc}
-\frac{1}{T_{x}} & 0 & \frac{k_{i}}{T_{x}} \\
\frac{1}{T_{m}} & -\frac{1}{T_{m}} & 0 \\
0 & 0 & -\frac{1}{T_{i}}
\end{array}\right]\left[\begin{array}{l}
I \\
X \\
S
\end{array}\right]+\left[\begin{array}{l}
0 \\
0 \\
\frac{1}{T_{i}}
\end{array}\right] U(t)} \\
& {\left[\begin{array}{c}
\dot{G} \\
\dot{Y}
\end{array}\right]=\left[\begin{array}{cc}
-\frac{1}{T_{y}} & \frac{1}{T_{G}} \\
\frac{K_{y}}{T_{y}} & -\frac{K_{y}}{T_{y}}\left(\frac{K_{y}}{T_{y}}+k_{i} X(t)\right.
\end{array}\right]\left[\begin{array}{l}
G \\
Y
\end{array}\right]}
\end{aligned}
$$

According to medical analysis the hepatic balance equations was designed to reduce the effective of meal (disturbance) (6)

$$
\begin{aligned}
& q_{A}=\left\{\begin{array}{c}
0.88 \quad \text { if } e_{A} \geq 0.88 \\
e_{A} \text { if } 0 \leq e_{A} \leq 0.88 \\
0
\end{array}\right. \\
& q_{g}=0.06 G-0.25 \\
& q_{f=\left\{\begin{array}{l}
q_{g} \\
0
\end{array} \text { if } \quad q_{g} \geq 0.25\right.} \\
& q_{c}=0.25 E_{g}+q_{g}
\end{aligned}
$$


$e_{m}=0.87+\tanh 0.0045(G-175)$

$e_{f}=0.11 e_{m}$

From the constraints system output, the assumed input and output are given as known parameter to the constraints system identification toolbox. It provides the best appropriate transfer function by using the adaptive tuning method to get the values of poles and zeroes and linearized the constraints system which is shown in Figure 3. The achieved system model is used to linearise the constraints system and the model predictive is designed to simulate the system as presented in Figure 4.

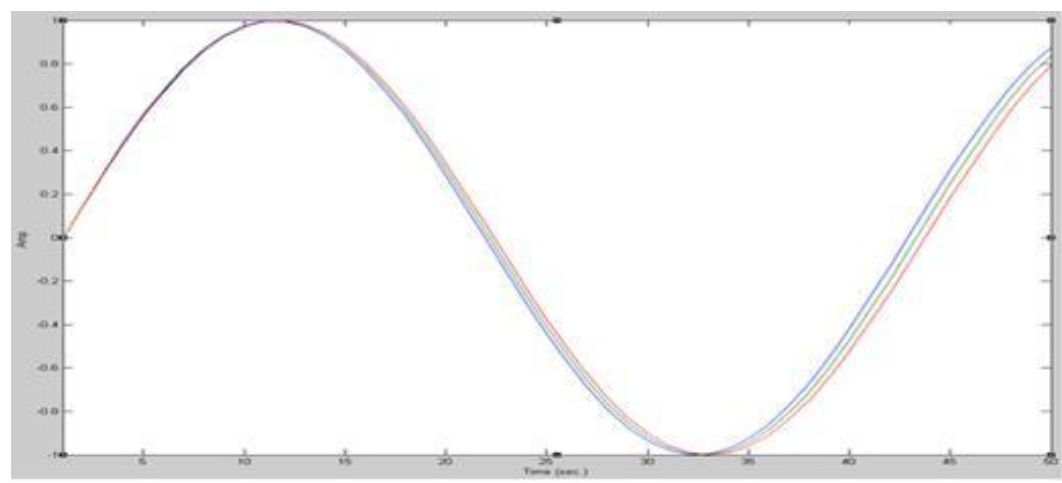

Figure 3. Measured and simulated system output

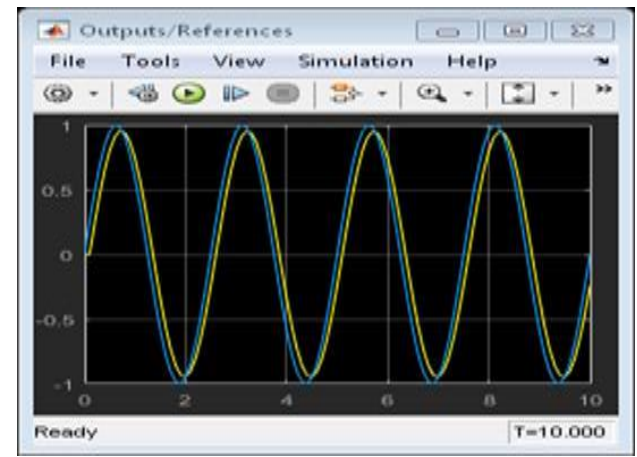

Figure 4. Input state (insulin) to output state (blood glucose) for plant

After finding the traditional (PID) controller, the plant is obviously checked and estimates the performance. However, the settling time taken is infinite and the process is not stable for the estimated output. While, when the RMPC is connected vice PID controller. The RMPC that have been designed the controlled variables and model output based on MPC controller toolbox. The inherent designed RMPC controller had model which is shown in Figure 5. RMPC can increase system speed and estimation accuracy. This technique gives a fairly proper estimate of the speed and is robust to any parameter variation.

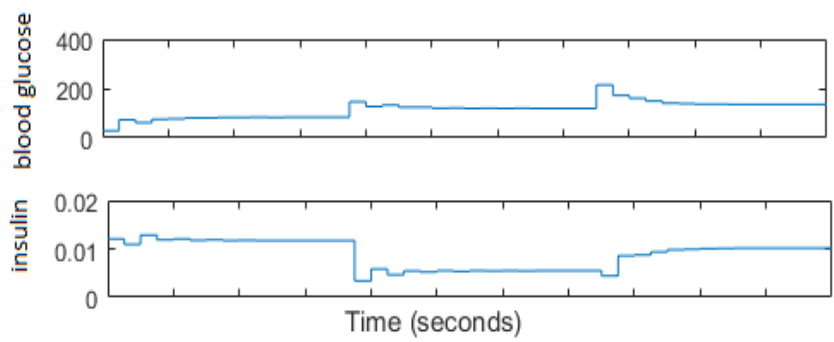

Figure 5. RMPC output for insulin and blood glucose states 


\section{CONCLUSIONS}

In this paper, we described RMPC based on a linearized form of constraints system. The state is increased with an inserted meal (disturbance) to ensure a state control of the blood glucose. We use MPC techniques to calculate insulin quantity in the cases where the insulin sensitivity reductions and in the case where the any meal (disturbance) is taken. The simulation results presented in this paper show that the designed RMPC is more effective, it has faster settling times. The uncertain parameter in input and its effect on the different factors of the real-time system are also measured. The designed RMPC not only considered of the unexpected disturbances in system, however also recover the certain parameters to the desired values, hence presenting the robustness in performance.

\section{REFERENCES}

[1] Boiroux, Dimitri; Finan, Daniel Aaron, "Strategies for glucose control in people with type 1 diabetes," In Proceedings of the 18th World Congress of the International Federation of Automatic Control (IFAC) DOI: 10.3182/20110828-6-IT-1002.03714,2011.

[2] Gianni Marchetti, Massimiliano Barolo, Lois Jovanovič, “A feedforward-feedback glucose control strategy for type 1 diabetes mellitus," Elsevier, Journal of Process Control, Volume 18, Issue 2, February 2008, Pages 149-162.

[3] Mojgan Esna-Ashari, Maryam Zekri, Masood Askari, "Predictive Control of the Blood Glucose Level in Type I Diabetic Patient Using Delay Differential Equation Wang Model," Journal of Medical Signals and Sensors, 2017 Jan-Mar; 7(1): 8-20.

[4] Lukas Ortmann, Dawei Shi, Eyal Dassau, "Gaussian Process-Based Model Predictive Control of Blood Glucose for Patients with Type 1 Diabetes Mellitus," arXiv:1707.09948v1 [cs.SY] 31 Jul 2017.

[5] C. Toffanin, R. Visentin, M. Messori, F. Di Palma, L. Magni, and C. Cobelli, "Towards a run-to-run adaptive artificial pancreas: In silico results," IEEE Trans. Biomed. Eng., 2017.

[6] B. J. Misgeld, P. G. Tenbrock, and S. Leonhardt, "Reduced-order filtering for insulin sensitivity estimation under external disturbances," in Amer. Control Conf. IEEE, 2017, pp. 1444-1449.

[7] C. D. Man M. Camilleri C. Cobelli, "A system model of oral glucose absorption: Validation on gold standard data," IEEE Trans. Biomed. Eng., vol. 53 no. 12 pp. 2472-2478 Dec. 2006.

[8] F. Chee T. Fernando, "Closed-Loop Control of Blood Glucose," Germany Berlin: Springer-Verlag 2007.

[9] A. Colosimo, "Medical Data Analysis," $3^{\text {rd }}$ international Symposium, ISMDA 2002, Rome, Italy, 2002 